\title{
Guidelines on the management of renal cyst disease
}

\author{
Thomas F. Whelan, MD, FRCSC
}

Can Urol Assoc J 2010;4(2):98-9

\section{Introduction}

Renal cysts are a common finding on routine radiological studies. As such, patients are often referred to urologists for their opinion regarding potential intervention and follow-up.

Autopsy studies in patients over the age of 50 reveal greater than a $50 \%$ chance of having at least one simple renal cyst. ${ }^{1}$ In 1983, using early computed tomography (CT) scan technology, renal cysts were discovered in $33 \%$ of patients in the same age group. ${ }^{2}$ Today, ultrasound and cross-sectional imaging studies are frequently used to aid in obtaining the diagnosis of abdominal complaints. With improved technology and newer generation diagnostic equipment, renal masses are more frequently identified than 25 years ago. ${ }^{3}$

\section{Methods}

The Canadian Urological Association Guidelines Committee has reviewed the literature using a MEDLINE search of the English language.

\section{Definition}

Renal cysts, in general, may be classified as "simple" or "complex." "Simple" cysts are best defined using sonographic criteria. These include: (1) absence of internal echoes, (2) posterior enhancement, (3) round/oval shape and (4) sharp, thin posterior walls. ${ }^{4}$ When all of the criteria are met, the cyst is benign and no follow-up is required. The difficulty arises when cysts do not meet the rigid characteristics of the "simple" definition. Therefore, clinicians need to rely on a rapid, safe and accurate system to identify benign versus malignant masses and ultimately have the guidance on nonsurgical or surgical treatment options.

\section{The Bosniak classification of renal cysts}

The Bosniak renal cyst classification system was initially reported in 1986, using CT scan findings. ${ }^{5}$ Although other imaging modalities are frequently used in the evaluation of renal masses, such as ultrasound and magnetic resonance imaging (MRI), CT scan (with and without contrast enhancement), remains the primary diagnostic technique. ${ }^{6}$

Ultrasonography is helpful for simple cyst identification, but provides limited information with increasingly complex renal cysts and solid masses. However, MRI can be helpful with increasingly complex cyst identification. Recent developments with MRI scanning allow shorter breath holds and increased contrast resolution with gadoliniumenhanced images. As such, the cysts may be characterized in greater detail, compared with CT scan. The MRI may demonstrate poorly identified septa on CT scan and show enhancements that are not otherwise clearly perceived. Also, MRI may differentiate between hemorrhagic cysts and solid enhancing masses. ${ }^{7}$

The Bosniak system consists of four categories based on triphasic $\mathrm{CT}$ findings, ranging from simple to complex cysts (Table 1). Category I cysts have no malignant potential and, as such, no follow-up is required. However, there is a large difference in potential malignant risk, between category II and category III. These are $0 \%$ to $5 \%$ and about $50 \%$, respectively. To clarify this further, a subcategory of II was developed, IIF (for "follow-up"). Category IIF identifies the category II cyst which was slightly more complicated, but not necessarily suspicious enough to warrant surgical exploration. Category IIF includes cysts which have multiple thin septa, slight wall-thickening without measurable contrast enhancement. They may have calcification, including thick, nodular or irregular calcification. Ultimately, $95 \%$ of category IIF cysts are proven to be nonmalignant. ${ }^{8}$ While the importance of calcification has diminished over the years since the original classification, enhancement with CT contrast has not. Any mass studied with CT thin slice scanning, that increases between 10 to 20 Hounsfield units (i.e., Category III and IV), is a renal cell carcinoma until proven otherwise. $^{7}$ 
Table 1. The Bosniak classification of renal cystic disease

\section{Category I: Malignant risk less than 1\%; no follow-up required}

- uncomplicated, simple benign cyst

- anechoic, posterior enhancement (through transmission), round or oval shape, thin, smooth wall

- homogeneous water content, sharp delineation with the renal parenchyma, no calcification, enhancement or wall-thickening

Category II: Malignant risk less than 3\%; no follow-up required

Cystic lesion with some abnormal radiological features

$-<1 \mathrm{~mm}$ septations (hairline thin)

- fine calcifications within the septum or wall

$-<3 \mathrm{~cm}$ in diameter

- hyperdense cysts (>20 Hounsfield units)

Category IIF: Malignant risk 5-10\%; follow-up recommended

Cystic lesion with increased abnormal findings

- multiple thin septum

- septa thicker than hairline or slightly thick wall

- calcification, which may be thick

- intrarenal, $>3 \mathrm{~cm}$

- no contrast enhancement

Category III: Malignant risk 40-60\%; surgical excision recommended More complicated

- uniform wall thickening/nodularity

- thick/irregular calcification

- thick septa

- enhances with contrast

Category IV: Malignant risk greater than $\mathbf{8 0} \%$; surgical excision recommended

- large cystic components

- irregular margins/prominent nodules

- solid enhancing elements, independent of septa

\section{Summary}

There are no randomized controlled trials with regards to follow-up or management of cystic renal masses, as such, the recommendations are primarily expert opinion.

At this time, category I and II renal cysts, do not require further imaging or follow-up. Patients in Category IIF, because of the approximate $5 \%$ malignant risk, do require periodic imaging. (There is no consensus or evidence based inter- val determined for follow-up imaging.) Combination of ultrasound and MRI should be considered as follow-up for Bosniak IIF and reduces the lifetime radiation dose (once the lesion has been characterized by triphasic CT scan) in patients younger than 50 years. For Category III (50\% malignant risk) and category IV (75\% to $90 \%$ malignant risk), surgical excision is recommended. ${ }^{9-13}$ (Level 3 evidence, Grade B recommendation). Although MRI may add further information, it should be used as an adjunct to CT scans in difficult cases (Level 4 evidence, Grade $C$ recommendation).

*St. John, NB; Dalhousie University, Halifax, NS

Competing interests: None declared.

This paper has been peer-reviewed.

\section{References}

1. Kissane JM. The morphology of renal cyst disease. Perspect Nephrol Hypertens 1976;4:31-63.

2. Tada S, Yamagishi J, Kobayashi $\mathrm{H}$, et al. The incidence of simple renal cyst by computed tomography. Clin Radiol 1983;34:437-9.

3. Minor LD, Picken MM, Campbell SC. Benign renal tumors. In: AUA Update Series; vol. 22. Houston, TX; 2003:170-1

4. Weber, TM. Sonography of benign renal cystic disease: Ultras Clin 2006;1:15-24.

5. Bosniak MA. The current radiological approach to renal cysts. Radio 1986;158:1-10.

6. Israel GM, Bosniak MA. An update on the Bosniak renal cyst classification system. Urology 2005;66:484-8.

7. Prasad SR, Dalrymple NC, Surabhi VR. Cross-sectional imaging evaluation of renal masses. Radio Clin 2008;46: 95-111.

8. Israel GM, Bosniak MA. Follow up CT scan for moderately complex cystic renal masses (Bosniak category II F). Am J Roentgenol 2003;181:627-33.

9. Aronson S, Frazier HA, Baluch JD, et al. Cystic renal masses: usefulness of the Bosniak classification. Urol Radiol 1991;13:83-90.

10. Siegel CL, McFarland EG, Brink JA, et al. CT of cystic renal masses: analysis of diagnostic performance and interobserver variation. AJR Am J Roentgenol 1997;169:813-8.

11. Koga S, Nishikido M, Inuzuka $S$, et al. An evaluation of Bosniak's radiological classification of cystic renal masses. BJU Int 2000;86:607-9.

12. Israel GM, Bosniak MA. Calcification in cystic renal masses: Is it important in diagnosis? Radiology 2003;226:47-52.

13. Curry NS, Cochran ST, Bissada NK. Cystic renal masses: accurate Bosniak classification requires adequate renal CT. AJR Am J Roentgenol 2000;175:339-42.

Correspondence: Dr. Thomas Whelan, PO Box 2100, Ste E-3D North, St. John, NB E2L 4L2; fax: 506-648-6894; thomas.whelan@horizonnb.ca 